ERRATUM

\title{
Mutations of CXorf6 are associated with a range of severities of hypospadias
}

Nicolas Kalfa ${ }^{1,2}$, Benchun Liu ${ }^{1}$, Ophir Klein ${ }^{3}$, Francoise Audran ${ }^{2}$, Ming-Hsieh Wang ${ }^{1}$, Cao Mei ${ }^{1}$, Charles Sultan ${ }^{2}$ and Laurence S Baskin ${ }^{1}$

${ }^{1}$ Department of Pediatric Urology, Center for the Study and Treatment of Hypospadias, Children's Medical Center, University of California San Francisco, 400 Parnassus Avenue, A 640, San Francisco, California 94143, USA, ${ }^{2}$ Service d'Hormonologie, Hôpital Lapeyronie, CHU Montpellier, Montpellier, France and ${ }^{3}$ Department of Orofacial Sciences and Pediatrics, Institutes of Human Genetics and Regeneration Medicine, University of California San Francisco, San Francisco, California, USA

(Correspondence should be addressed to L S Baskin; Email: lbaskin@urology.ucsf.edu)

The authors and journal apologize for an error in the above paper above which appeared in 159 (4) 453-458. The third author's name should have appeared as Ophir Klein, as shown correctly above.

European Journal of Endocrinology 161511 